\title{
Synthesis of photoresponsive cholesterol-based azobenzene organogels: dependence on different spacer lengths
}

\author{
Yuchun Ren, Bin Wang ${ }^{*}$ and Xiuqing Zhang
}

\author{
Full Research Paper \\ Address: \\ Chemical Synthesis and Pollution Control Key Laboratory of Sichuan \\ Province of China, China West Normal University, Nanchong 637009, \\ China \\ Email: \\ Bin Wang* - cwnuwangb@126.com \\ * Corresponding author \\ Keywords: \\ azobenzene; cholesterol; organogel; photoresponsive
}

Beilstein J. Org. Chem. 2015, 11, 1089-1095.

doi:10.3762/bjoc.11.122

Received: 16 January 2015

Accepted: 08 June 2015

Published: 29 June 2015

Associate Editor: H. Ritter

(c) 2015 Ren et al; licensee Beilstein-Institut.

License and terms: see end of document.

\begin{abstract}
A series of azobenzene-cholesterol organogel compounds $\left(\mathbf{M}_{\mathbf{0}}-\mathbf{M}_{\mathbf{1 2}}\right)$ with different spacers were designed and synthesized. The molecular structures were confirmed by ${ }^{1} \mathrm{H}$ NMR and ${ }^{13} \mathrm{C}$ NMR spectroscopy. The rapid and reversible photoresponsive properties of the compounds were investigated by UV-vis spectroscopy. Their thermal phase behaviors were studied by DSC. The length of the spacer plays a crucial role in the gelation. Compound $\mathbf{M}_{\mathbf{6}}$ is the only one that can gelate in ethanol, isopropanol and 1-butanol and the reversible gel-sol transitions are also investigated. To obtain visual insight into the microstructure of the gels, the typical structures of the xerogels were studied by SEM. Morphologies of the aggregates change from flower-like, network and rod with different sizes. By using IR and XRD characterization, it is found that intermolecular H-bonding, the solvents and van der Waals interaction are the main contributions to the specific superstructure.
\end{abstract}

\section{Introduction}

In the past few decades, low molecular mass organic gelators (LMOGs) have attracted increasing attention not only for basic self-assembly behavior but also for their potential application in areas such as templates [1], light harvesting [2], fluorescent scensing [3], etc. The driving force for the spontaneous formation of gel could be relatively non-covalent interactions such as $\pi-\pi$ stacking [4-7], hydrogen bonding [7-9], dipole-dipole [10], and van der Waals interactions [11,12]. A series of cholesterolbased gelators were reported [13-15]. These new classes of organogelator architectures have been systematically studied because of their reversible gel process and unique directional self-association through weak van der Waals interactions $[16,17]$. Up to now, numerous attempts have been made to develop novel supramolecular architectures, which can bring about new predictable gelation abilities for the construction of novel cholesterol-based gelators.

Azobenzene is one of the smartest molecules among all known photochromic compounds because of its $E / Z$ isomerization [18]. Based on this, photomechanical soft materials containing azobenzene have been utilized in supramolecular systems [19]. Examples of several cholesterol-linked azobenzenes with 
gel-sol reversible changes also have been studied [20-23]. R. Zentel found that the photoinduced reversible gel-sol changes between isotropic and anisotropic gels of a class of semicarbazide-azobenzene-based gelators [24]. A new multistimuli photoresponsive organogel containing azobenzene groups was designed and studied by D. Zhu and his co-workers [25]. Q. Zhang synthesized a series of new symmetric dicholesterol-linked gelators [26]. Upon UV-vis irradiation of the gels, a reversible gel-sol transition occurred by breaking and reforming of van der Waals interactions. The cholesterol-substituted diacetylenic polymerized gels exhibit enhanced stability upon thermo- or photo-stimuli [27]. Although similar compounds have been discussed, it is still not very clear about the relationship between the spacer length and the gelation ability of gelators. Herein we synthesized a series of compounds with different spacers between cholesteryl and azobenene units (Scheme 1). In these compounds, $\mathbf{M}_{\mathbf{6}}$ is the only one that can gelate in ethanol, isopropanol and 1-butanol. It is found that intermolecular H-bonding, the solvents and van der Waals interaction are the main contributions to the specific superstructure.

\section{Results and Discussion}

To investigate the photoresponsiveness of these compounds, the dilute THF solution of compounds were irradiated by UV and visible light. Figure 1a shows an obvious increase in the peak at $258 \mathrm{~nm}$ and a concurrent decrease in the strong peak at $358 \mathrm{~nm}$. The photostationary state was attained after 94 min of irradiation and the spectral changes are attributable to the trans-to-cis isomerization under UV light irradiation. Further visible light $(450 \mathrm{~nm})$ irradiation on the $c i s$-solution led to an increase in the absorption intensity at $358 \mathrm{~nm}$ and a decrease around $258 \mathrm{~nm}$, and the original spectrum recovered by the reverse cis-to-trans isomerization (Figure 1b). The absorption band at $358 \mathrm{~nm}$ corresponding to the $\pi-\pi^{*}$ transition of azobenzene increased, respectively. It should be emphasized that such reversible spectral transformation could be repeated several times. The transto-cis isomerization of the azobenzenze unit had been demonstrated. Compounds $\mathbf{M}_{2}, \mathbf{M}_{5}, \mathbf{M}_{6}$, and $\mathbf{M}_{12}$ have the same recoverable photoresponsive properties as $\mathbf{M}_{\mathbf{0}}$. Their UV-vis spectra were shown in Supporting Information File 1, Figures S1-S4. $\mathbf{M}_{\mathbf{3}}$ and $\mathbf{M}_{10}$ have the photochromic properties.<smiles>CC(C)CCC[C@H](C)[C@H]1CCC2C3CC=C4C[C@@H](OCCO)CC[C@]4(C)C3CC[C@]2(C)C1CO</smiles>

cholesterol

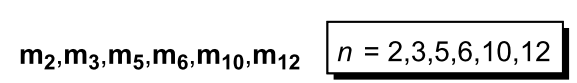<smiles>COc1ccc(/N=N/c2ccc(C(=O)O)cc2)cc1</smiles><smiles>COC(=O)c1ccc(N=Nc2ccc(OC)cc2)cc1</smiles><smiles></smiles> 

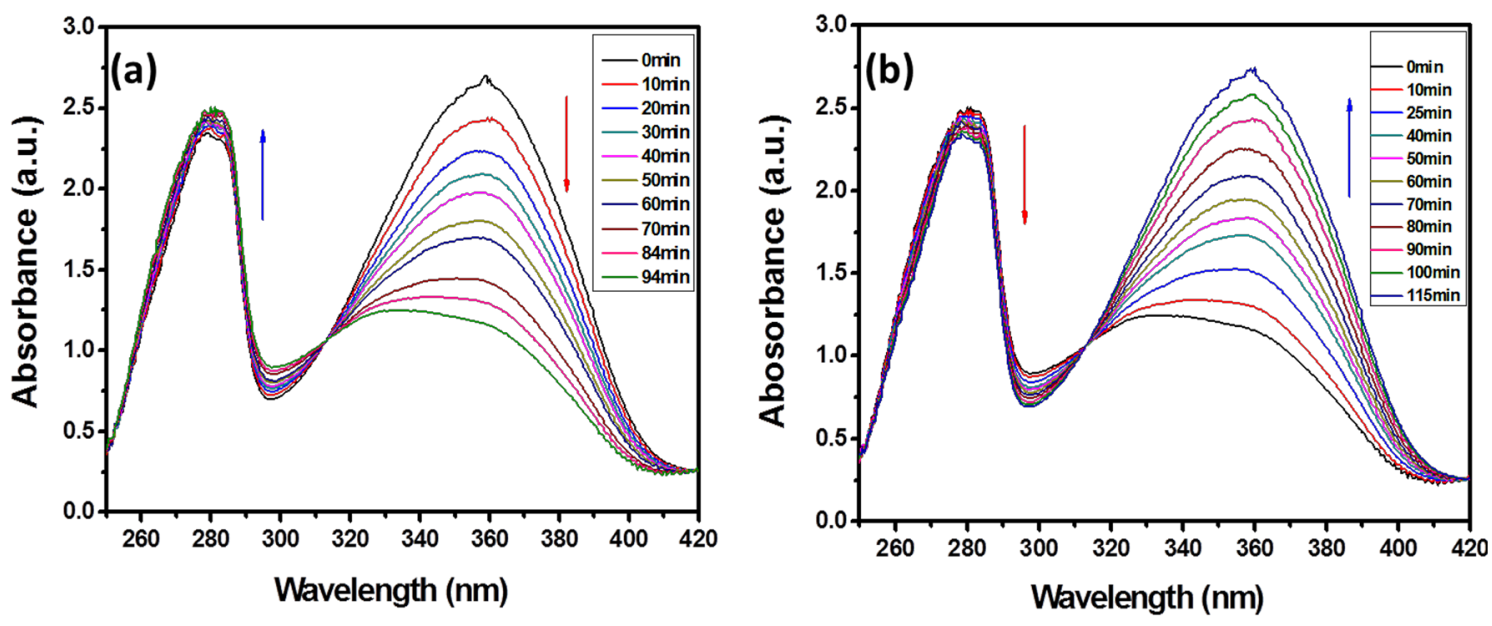

Figure 1: Changes in the absorption over time in the UV-vis spectra of dilute THF solution of $M_{0}$ : (a) upon UV-light irradiation $(\lambda=365 \mathrm{~nm})$ and (b) upon visible-light irradiation $(\lambda=450 \mathrm{~nm})$ of the solution obtained after irradiation of $365 \mathrm{~nm}$.

\section{Liquid crystal properties of the synthesized compounds}

The structures of these compounds are shown in Scheme 1. The thermal behaviors of the seven compounds were analyzed by differential scanning calorimetry (DSC). The DSC curves of the cholesterol-based supramolecular complexes are shown in Figure 2 and phase transition temperatures are listed in Table 1. The DSC thermogram of $\mathbf{M}_{\mathbf{0}}$ presented three peaks at 158, 211 and $249^{\circ} \mathrm{C}$ on heating, corresponding to the crystal to crystal transition, crystal to mesophase transition and mesophase to isotropic transition. On the cooling run, DSC curves presented isotropic phase to mesophase, mesophase to crystal and crystal to crystal at 190,155 and $126^{\circ} \mathrm{C}$. Each curve of compounds $\mathbf{M}_{2}, \mathbf{M}_{\mathbf{6}}$ and $\mathbf{M}_{10}$ displayed a single endothermic melting peak. The heating DSC curves of $\mathbf{M}_{\mathbf{3}}, \mathbf{M}_{\mathbf{5}}$ and $\mathbf{M}_{\mathbf{1 2}}$ contained two endothermic peaks. The peaks at lower temperature were due to

\begin{tabular}{|c|c|c|}
\hline sample & heating $\left({ }^{\circ} \mathrm{C}\right)$ & cooling $\left({ }^{\circ} \mathrm{C}\right)$ \\
\hline $\mathbf{M}_{0}$ & K $158 K_{2} 211$ N 249 I & I 190 N $155 K_{1} 126 K_{2}$ \\
\hline$M_{2}$ & K 92 I & I $59 \mathrm{~K}$ \\
\hline$M_{3}$ & K 164 N 220 I & I 224 N 213 K \\
\hline$M_{5}$ & K 120 N 197 I & I 193 N 83 K \\
\hline$M_{6}$ & K 78 I & - \\
\hline $\begin{array}{l}M_{10} \\
M_{12}\end{array}$ & $\begin{array}{l}\text { K } 90 \text { I } \\
\text { K } 75 \text { N } 120 \text { I }\end{array}$ & $\begin{array}{l}\text { I } 54 \mathrm{~K} \\
\text { I } 120 \text { N } 42 \mathrm{~K}\end{array}$ \\
\hline
\end{tabular}

$\mathrm{K}$ : crystalline state, $\mathrm{N}$ : nematic phase, I: isotropic liquid.

the crystal to mesophase transitions, and the second peaks displayed the mesophase to isotropic transitions. The DSC thermogram of $\mathbf{M}_{\mathbf{3}}, \mathbf{M}_{\mathbf{5}}$ and $\mathbf{M}_{\mathbf{1 2}}$ were observed two exothermic

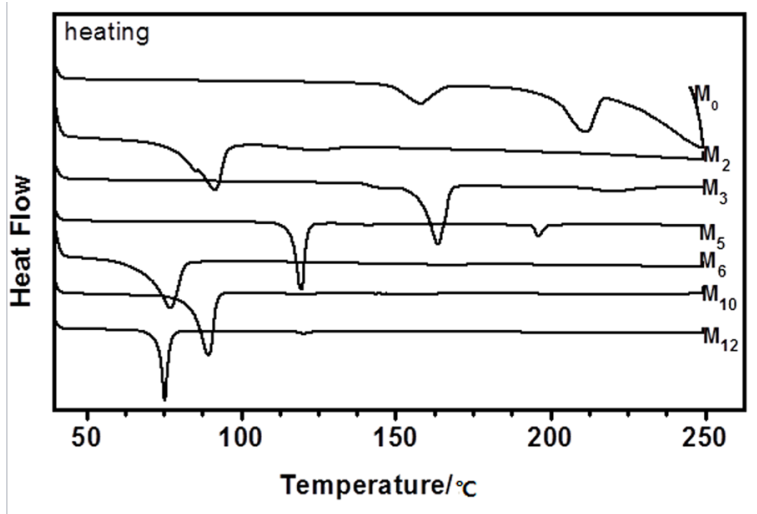

(a)

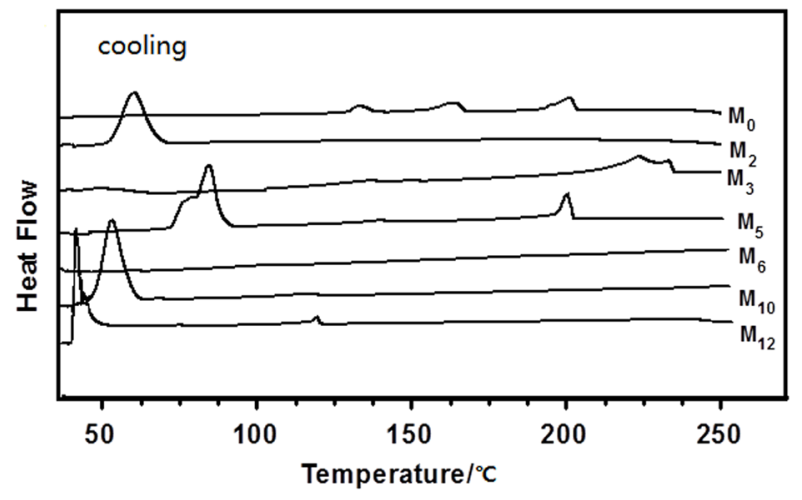

(b)

Figure 2: DSC thermogram of the compounds $\mathbf{M}_{\mathbf{0}-\mathrm{n}}$ obtained on (a) heating and (b) cooling. 
peaks on cooling process, which indicated the transitions from isotropic phase to mesophase at higher temperature and mesophase to crystal transitions at lower temperature

\section{Gelation behaviors}

The gelation behaviors of the seven compounds were tested in 22 solvents at room temperature and the results were listed in Table 2. Among the compounds examined, $\mathbf{M}_{\mathbf{6}}$ is the only one that forms a gel in ethanol, 1-butanol and isopropanol, while the other compounds cannot gelate in any solvent. Generally speaking, the gels were formed by a heating and cooling cycle process. Examination of the data shown in the table reveals that

Table 2: Gelation performances of the seven cholesteryl derivatives at room temperature.

\begin{tabular}{|c|c|c|c|c|c|c|c|}
\hline Solvent & $\mathbf{M}_{0}$ & $\mathbf{M}_{2}$ & $\mathbf{M}_{3}$ & $\mathbf{M}_{5}$ & $M_{6}$ & $M_{10}$ & $\mathbf{M}_{12}$ \\
\hline Methanol & 1 & I & I & I & 1 & 1 & I \\
\hline Ethanol & I & 1 & I & 1 & G & 1 & I \\
\hline 1-Propanol & 1 & 1 & I & I & I & I & 1 \\
\hline 1-Butanol & 1 & I & I & $\mathrm{P}$ & G & I & I \\
\hline 1-Pentanol & I & I & I & I & I & I & I \\
\hline 1-Octanol & 1 & $\mathrm{P}$ & I & 1 & 1 & I & I \\
\hline 1,4-Butanediol & 1 & 1 & I & I & I & 1 & I \\
\hline Isopropanol & 1 & 1 & I & I & G & I & I \\
\hline Acetone & 1 & $\mathrm{P}$ & I & $\mathrm{P}$ & $S$ & 1 & $\mathrm{P}$ \\
\hline DMSO & I & I & I & I & I & I & I \\
\hline DMF & I & $\mathrm{P}$ & 1 & 1 & $S$ & I & I \\
\hline EtOAc & I & $S$ & I & $\mathrm{P}$ & $S$ & $\mathrm{P}$ & $\mathrm{P}$ \\
\hline Kerosene & I & $\mathrm{P}$ & I & I & I & $\mathrm{P}$ & $S$ \\
\hline Benzene & $\mathrm{S}$ & $S$ & $\mathrm{~S}$ & $S$ & $S$ & $S$ & $S$ \\
\hline Toluene & $\mathrm{S}$ & $S$ & $\mathrm{~S}$ & $S$ & $S$ & $S$ & $S$ \\
\hline Xylene & $S$ & $S$ & $\mathrm{~S}$ & $S$ & $S$ & $S$ & $S$ \\
\hline THF & $S$ & $S$ & $\mathrm{~S}$ & $S$ & $S$ & $S$ & $S$ \\
\hline$n$-Hexane & I & $\mathrm{P}$ & I & $\mathrm{P}$ & $\mathrm{P}$ & $\mathrm{P}$ & $\mathrm{P}$ \\
\hline Dichloromethane & $S$ & $S$ & $\mathrm{~S}$ & $S$ & $S$ & $S$ & $S$ \\
\hline Chloroform & $S$ & $S$ & $S$ & $S$ & $S$ & $S$ & $S$ \\
\hline Tetrachloromethane & $S$ & $S$ & $\mathrm{~S}$ & $S$ & $S$ & $S$ & $S$ \\
\hline Distilled water & I & I & I & I & I & I & I \\
\hline
\end{tabular}

G: turbid gel; S: solution; P: precipitate; I: insoluble. the change of spacer length in the molecular skeleton has a significant effect on the gelation abilities of the compounds. To investigate the photoresponsiveness of the three gels, we performed the photoirradiation of gels using a UV lamp. The formed gels at room temperature were in an opaque state. Furthermore, the gels with both solvents are very stable for months of storage in a closed container, and no significant changes can be found. However, upon UV irradiation, the gel to sol phase transition became transparent as a result of trans-tocis isomerization. Moreover, the solution has a tendency to reverse the sol to gel phase transition under visible irradiation. The corresponding visual images are shown in Figure 3.

\section{Scanning electron microscopy studies}

To obtain a visual insight into the morphology of the aggregation mode, the typical structures of the xerogels prepared by the freeze-drying of the three gels in different solvents were studied by SEM (Figure 4). With the reference to the images, it can be clearly seen that the structures of the three gelators are significantly different from each other. The morphologies of the aggregates change from flower-like to network and rod with different sizes. Obviously, the gel from ethanol mainly shows a similar flower-like morphology. When we focused on the isopropanol xerogel, we could see that there are many regularly three-dimensional network structures. In 1-butanol, the organogelator molecules in the gel phase were self-assembled into tightly staked rods. Different solvents caused a sharp difference between these morphologies of the xerogels.

\section{The FTIR spectroscopy}

It should be emphasized that the organogels achieved through strength and directionality of hydrogen bonding. Further information about the intermolecular interactions of these gels was obtained from the FTIR spectroscopy. Figure 5 shows the IR spectra of $\mathbf{M}_{\mathbf{6}}$ powder and its gels in ethanol, isopropanol and 1-butanol. Gels show two peaks which located at 3740 and $3672 \mathrm{~cm}^{-1}$, characteristic of the hydrogen bonding $\mathrm{O}-\mathrm{H}$ stretching vibration. As for the powder, the absorption bands cannot be found in the same vibrational area. The peak
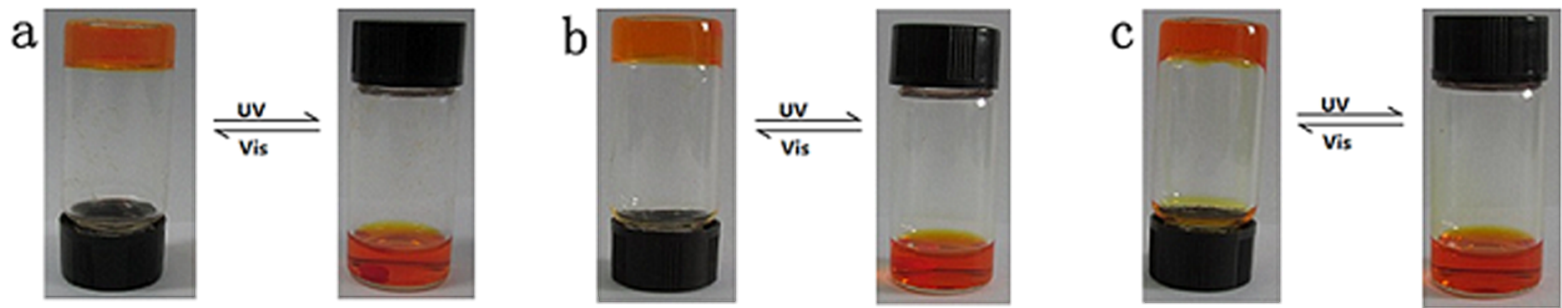

Figure 3: Photographic images of the $\mathbf{M}_{\mathbf{6}}$ sol-gel transition behavior after $\mathbf{U V}$ or visible light irradiation in different solution (a) ethanol, (b) isopropanol and (c) 1-butanol. 

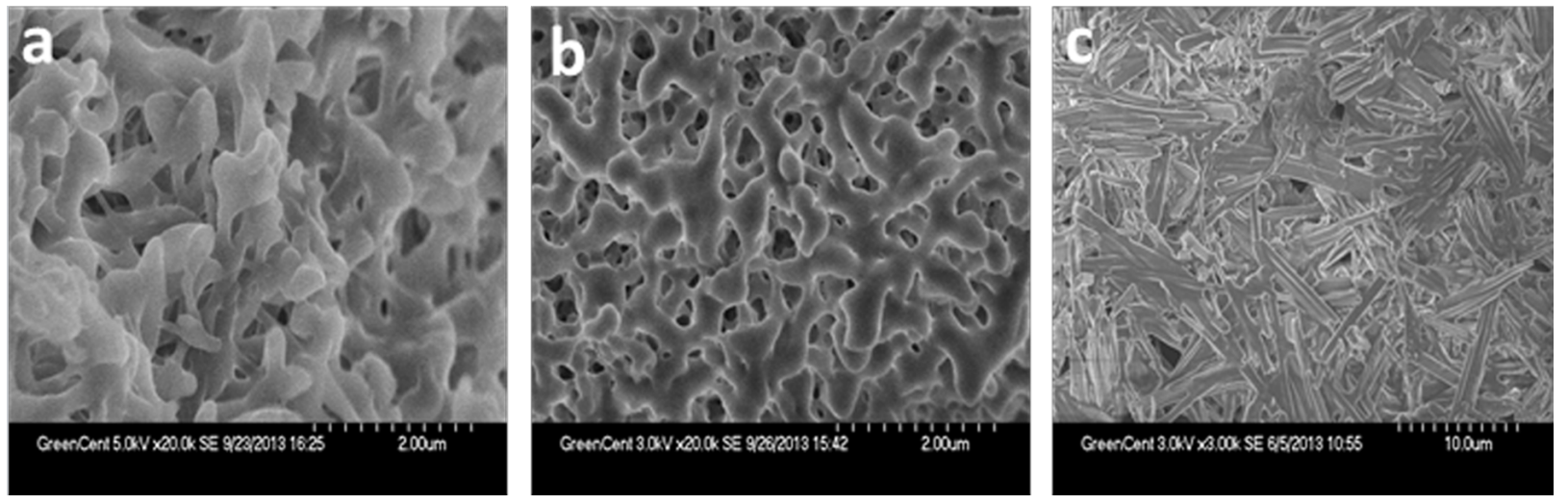

Figure 4: SEM images of xerogels formed with $\mathbf{M}_{6}$ from solution: (a) ethanol, (b) isopropanol and (c) 1-butanol.

observed at $3437 \mathrm{~cm}^{-1}$ can be assigned to the intermolecular association hydrogen stretching vibration. However, the band of ethanol, isopropanol and 1-butanol shifted to 3438, 3439 and $3440 \mathrm{~cm}^{-1}$, respectively. The comparison of the IR spectra of powder and gels indicated the hydrogen-bond interaction in the gels. The bands of the asymmetric and symmetric $\mathrm{CH}_{2}$ stretching vibration of $\mathbf{M}_{\mathbf{6}}$ powder emerged at 2935 and $2852 \mathrm{~cm}^{-1}$, while, in the ethanol gel, they shifted to 2934 and $2858 \mathrm{~cm}^{-1}$, in isopropanol gel, they shifted to 2934 and $2862 \mathrm{~cm}^{-1}$, in 1-butanol, they shifted to 2933 and $2859 \mathrm{~cm}^{-1}$ The above mentioned FTIR spectral changes demonstrated sufficiently that van der Waals interaction between the alkyl chains plays an important role in the self-assembly process of organogels.

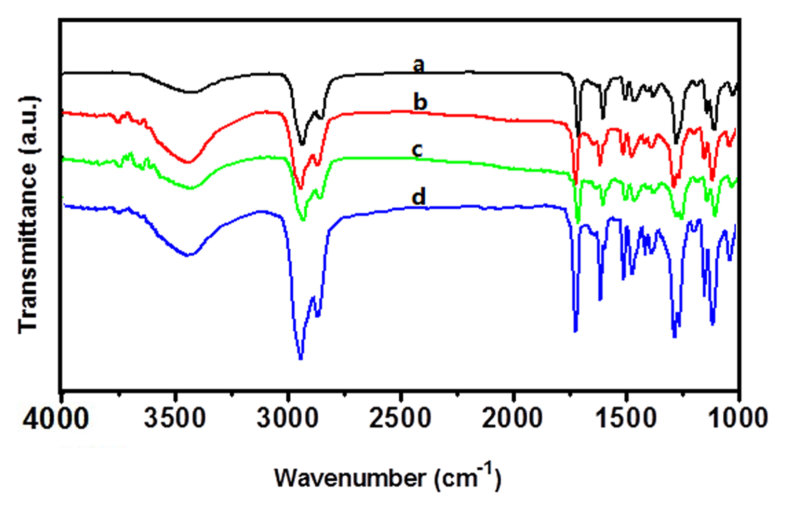

Figure 5: FTIR spectra of gels: (a) powder, (b-d) ethanol, isopropanol and 1-butanol xerogels, respectively.

\section{The XRD studies}

To reveal the detailed changes in gel molecular of $\mathbf{M}_{\mathbf{6}}, \mathrm{XRD}$ analyses were conducted and the results were displayed in Figure 6. The pattern of the xerogel obtained from the 1-butanol gel exhibited five peaks at $12.84,15.38,17.22$, and $20.44^{\circ}$, corresponding to $d$-spacing of $6.90,5.80,5.14,4.30$, and
$3.70 \mathrm{~nm}$, respectively. The $d$-spacing almost in ratio of 7:6:5:4, may due to the molecular arrangement with order or existence layers of the stacking between molecular. The last peak may relate to the periodic intermolecular distances at different direction and intermolecular separations. Two diffraction peaks at $17.32^{\circ}$ and $25.40^{\circ}$ were detected for the xerogels of another two solvents, corresponding to $d$ values of 5.10 and $3.50 \mathrm{~nm}$, respectively. The different self-assembly modes of gelator in various solvents cause the values changing. The XRD results described above also demonstrated that the molecular interaction and the solvents had a significant impact on the gel formation modes.

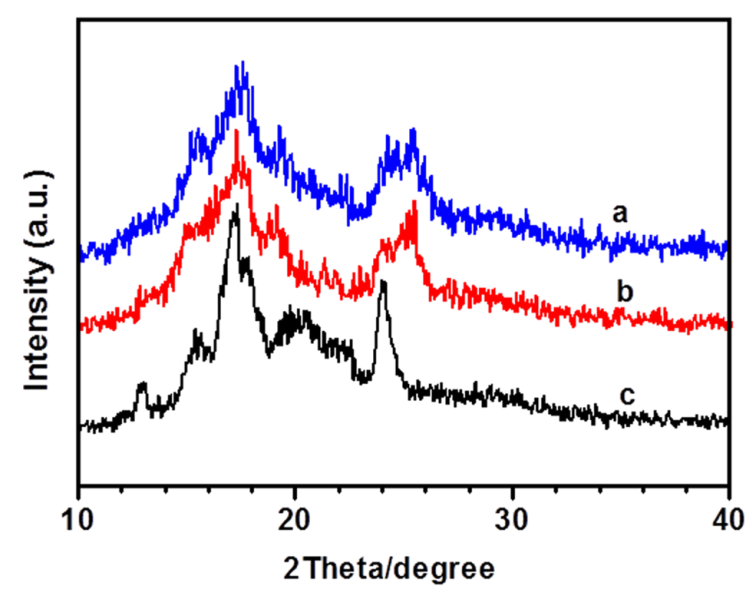

Figure 6: XRD patterns of xerogels. (a) ethanol, (b) isopropanol and (c) 1-butanol.

\section{Experimental \\ Materials}

The starting materials, thylene glycol, 1,3-propanediol, 1,5pentadiol, 1,6-hexanediol, 1,10-decanediol, 1,12-dodecanediol, cholesterol, 4-toluene sulfonyl chloride, phenol, 4-aminobenzoic acid, iodomethane, sodium nitrite, hydrochloric acid, 
sodium hydroxide, $N, N^{\prime}$-dicyclohexylcarbodiimide (DCC), 4-dimethylaminopyridine (DMAP), all were purchased from Sigma-Aldrich Chemicals (Shanghai, China). Other used reagents were commercially available and were distilled before used. The synthesis of the 4'-carboxy-4-methoxyazobenzene ultilized in this study was described elsewhere [28].

\section{General measurements}

NMR spectra were recorded with a Bruker-400 NMR spectrometer. Powder X-ray diffraction (XRD) measurements were carried out on a Rigaku D/max 2500PC (Japan)-type singlecrystal diffractometer using $\mathrm{Cu} \mathrm{K} \alpha$ radiation to determine the crystal structure. Infrared spectroscopy (IR) was measured on a Nicolet 6700 spectrometer from 4000 to $500 \mathrm{~cm}^{-1}$ at room temperature. The samples were prepared as $\mathrm{KBr}$ pellets. The morphology of the gel samples were examined by scanning electron microscopy (SEM, S5200, FEI Company) under an acceleration voltage of $30 \mathrm{kV}$. UV-vis absorption spectra were recorded using a spectrophotometer (UV-3600, Shimadzu, Japan). Differential scanning calorimetry (DSC) was carried out on a thermal analysis (TA) DSC-Q30 in nitrogen at a heating rate of $10 \mathrm{~K} / \mathrm{min}$.

\section{Synthetic procedures}

Synthesis of $\mathbf{m}_{\mathbf{2}}-\mathbf{m}_{\mathbf{1 2}} \cdot 3 \beta$-[(2-hydroxyethyl)oxy]cholest-5-ene $\left(\mathbf{m}_{2}\right)$ was synthesized similar to a previous report [29]. Cholesterol $15.00 \mathrm{~g}(38 \mathrm{mmol})$ in dry dichloromethane $(225 \mathrm{~mL})$ and dry triethylamine $(16.50 \mathrm{~mL}), p$-tosyl chloride $10.35 \mathrm{~g}$ (54 mmol) was added. A catalytic amount of DMAP was also added. The reaction mixture was then allowed to stir at $48{ }^{\circ} \mathrm{C}$ for $12 \mathrm{~h}$. The reaction mixture was washed sequentially with diluted $\mathrm{HCl}$ aqueous solution, saturated brine solution, and water. The organic phase was dried over anhydrous $\mathrm{Na}_{2} \mathrm{SO}_{4}$. The solvents were removed by vacuum evaporation and recrystallized using chloroform and methanol. The residue (7.0 g, $13 \mathrm{mmol}$ ) was dissolved in anhydrous dioxane $(60 \mathrm{~mL})$ and dry ethylene glycol (20 g, $0.32 \mathrm{~mol})$ was added. The mixture was refluxed at $110{ }^{\circ} \mathrm{C}$ for $4 \mathrm{~h}$. The solution was cooled and solvent was removed under vacuum. The white residue was dissolved in chloroform. The organic layer washed with $\mathrm{NaHCO}_{3}$, water, and brine; and dried over anhydrous $\mathrm{Na}_{2} \mathrm{SO}_{4}$. Finally, the product was purified by column chromatography over silica gel eluting with petroleum ether/ethyl acetate to yield $90 \%$ of $\mathbf{m}_{\mathbf{2}}$. The procedure used for the preparation of $\mathbf{m}_{\mathbf{3}}, \mathbf{m}_{\mathbf{5}}, \mathbf{m}_{\mathbf{6}}, \mathbf{m}_{\mathbf{1 0}}$, and $\mathbf{m}_{\mathbf{1 2}}$ are similar to that for $\mathbf{m}_{\mathbf{2}}$.

\section{4-Methoxy-4'-((cholesteryloxy)carbonyl)azo- benzene $\left(\mathbf{M}_{\mathbf{0}}\right)$}

A mixture of 4'-carboxy-4-methoxyazobenzene $(0.25 \mathrm{~g}$, $10 \mathrm{mmol})$, cholesterol $(0.38 \mathrm{~g}, 1 \mathrm{mmol})$, and DCC $(0.21 \mathrm{~g}$, $1 \mathrm{mmol})$, DMAP (0.06 g, $0.5 \mathrm{mmol})$ in $25 \mathrm{~mL} \mathrm{CH}_{2} \mathrm{Cl}_{2}$ was stirred at room temperature for $24 \mathrm{~h}$. The solvent was evaporated under vacuum. The product was purified by column chromatography over silica gel eluting with pure $\mathrm{CH}_{2} \mathrm{Cl}_{2}$, yield $70 \%$. Related compounds $\left(\mathbf{M}_{2}, \mathbf{M}_{3}, \mathbf{M}_{5}, \mathbf{M}_{6}, \mathbf{M}_{10}, \mathbf{M}_{12}\right)$ were synthesized by a similar method.

\section{Gelation test}

The potential gelator $(25 \mathrm{mg})$ in solvent $(1 \mathrm{~mL})$ was placed in a sealed test tube and the solution was heated in a water bath until the solid was dissolved completely. The solution was cooled to room temperature. If the gelator formed a gel at this stage, it was classified as "G". When the solution remained all the time, they were referred to as solutions (S). The hot clear solution systems, which precipitated when they are cooled down to room temperature, are donated as $\mathrm{P}$ (precipitation), respectively. Insoluble systems in which the potential gelator could not be dissolved even at the boiling point of the solvent were designated as insoluble (I).

\section{Conclusion}

In summary, we have synthesized seven cholesterol-based compounds with azobenzene groups. The compounds with different flexible alkyl spacers containing two, three, five, six, ten, or twelve carbon atoms are denoted as $\mathbf{M}_{2}-\mathbf{M}_{12}$, respectively. Upon UV-vis irradiation, these compounds can undergo a typical reversible trans-cis and cis-trans change due to the photo-isomerization of the azobenzene units. They possess recoverable photoresponsive properties. Their thermal phase behaviors have been investigated by DSC, indicating good liquid crystal properties. The dramatic change in the gelation behavior was produced by the different spacer lengths between cholesteryl and azobenzene. Compound $\mathbf{M}_{\mathbf{6}}$ is the only good gelator that can gelate in ethanol, isopropanol and 1-butanol. Based on the results of SEM, IR and XRD, it demonstrated that the solvents, intermolecular H-bonding, and van der Waals interaction affected the aggregation mode and morphology of gels. These results can be applied in fields of design and development of novel organogelators and soft matter.

\section{Supporting Information}

\section{Supporting Information File 1}

Spectroscopical and analytical data.

[http://www.beilstein-journals.org/bjoc/content/

supplementary/1860-5397-11-122-S1.pdf]

\section{Acknowledgements}

The work was financially supported by the Program for Scientific and Technological Innovative Team in Sichuan Provincial Universities of China (2010008), Key Project of Natural 
Science of Sichuan Educational Department of China (12ZA175), Chemical synthesis and Pollution Control Key Laboratory of Sichuan Province of China (CSCP2013-2), and University research and launch projects of China West Normal University in 2014 (14C003).

\section{References}

1. van Bommel, K. J. C.; Friggeri, A.; Shinkai, S. Angew. Chem., Int. Ed. 2003, 42, 980-999. doi:10.1002/anie.200390284

2. Sugiyasu, K.; Fujita, N.; Shinkai, S. Angew. Chem., Int. Ed. 2004, 43, 1229-1233. doi:10.1002/anie.200352458

3. Kartha, K. K.; Babu, S. S.; Srinivasan, S.; Ajayaghosh, A. J. Am. Chem. Soc. 2012, 134, 4834-4841. doi:10.1021/ja210728c

4. van Gorp, J. J.; Vekemans, J. A. J. M.; Meijer, E. W. J. Am. Chem. Soc. 2002, 124, 14759-14769. doi:10.1021/ja020984n

5. An, B.-K.; Lee, D.-S.; Lee, J.-S.; Park, Y.-S.; Song, H.-S.; Park, S. Y. J. Am. Chem. Soc. 2004, 126, 10232-10233. doi:10.1021/ja046215g

6. Kishimura, A.; Yamashita, T.; Aida, T. J. Am. Chem. Soc. 2005, 127, 179-183. doi:10.1021/ja0441007

7. Shao, H.; Parquette, J. R. Chem. Commun. 2010, 46, 4285-4287. doi:10.1039/c0cc00701c

8. de Jong, J. J. D.; Lucas, L. N.; Kellogg, R. M.; van Esch, J. H.; Feringa, B. L. Science 2004, 304, 278-281. doi:10.1126/science.1095353

9. Yagai, S.; Nakajima, T.; Kishikawa, K.; Kohmoto, S.; Karatsu, T.; Kitamura, A. J. Am. Chem. Soc. 2005, 127, 11134-11139. doi:10.1021/ja052645a

10. Mamiya, J.-i.; Kanie, K.; Hiyama, T.; Ikeda, T.; Kato, T. Chem. Commun. 2002, 1870-1871. doi:10.1039/b205072b

11. Suzuki, M.; Nigawara, T.; Yumoto, M.; Kimura, M.; Shirai, H.; Hanabusa, K. Org. Biomol. Chem. 2003, 1, 4124-4131. doi:10.1039/b308371c

12. Kramb, R. C.; Zukoski, C. F. Langmuir 2008, 24, 7565-7572. doi:10.1021/la800021h

13. Chen, Q.; Zhang, D.; Zhang, G.; Zhu, D. Langmuir 2009, 25, 11436-11441. doi:10.1021/la9016257

14. Lee, W.-K.; Kim, K.-N.; Achard, M. F.; Jin, J.-I. J. Mater. Chem. 2006, 16, 2289-2297. doi:10.1039/b516141j

15. Xu, F.; Wang, H.; Zhao, J.; Liu, X.; Li, D.; Chen, C.; Ji, J. Macromolecules 2013, 46, 4235-4246. doi:10.1021/ma400276u

16. Peng, J.; Liu, K.; Liu, J.; Zhang, Q.; Feng, X.; Fang, Y. Langmuir 2008, 24, 2992-3000. doi:10.1021/la703672u

17. Chen, P.; Lu, R.; Xue, P.; Xu, T.; Chen, G.; Zhao, Y. Langmuir 2009, 25, 8395-8399. doi:10.1021/la8035727

18. Xie, S.; Natansohn, A.; Rochon, P. Chem. Mater. 1993, 5, 403-411. doi:10.1021/cm00028a003

19. Barrett, C. J.; Mamiya, J.-i.; Yager, K. G.; Ikeda, T. Soft Matter 2007, 3, 1249-1261. doi:10.1039/b705619b

20. Murata, K.; Aoki, M.; Suzuki, T.; Harada, T.; Kawabata, H.; Komori, T.; Ohseto, F.; Ueda, K.; Shinkai, S. J. Am. Chem. Soc. 1994, 116, 6664-6676. doi:10.1021/ja00094a023

21. Jung, J. H.; Ono, Y.; Shinkai, S. Angew. Chem., Int. Ed. 2000, 39 , 1862-1865. doi:10.1002/(SICI)1521-3773(20000515)39:10<1862::AID-ANIE1862>3 .0.CO;2-3

22. Jung, J. H.; Shinkai, S.; Shimizu, T. Chem. Mater. 2003, 15, 2141-2145. doi:10.1021/cm0217912
23. Koumura, N.; Kudo, M.; Tamaoki, N. Langmuir 2004, 20, 9897-9900. doi:10.1021/la048334f

24. Deindörfer, P.; Davis, R.; Zentel, R. Soft Matter 2007, 3, 1308-1311. doi:10.1039/b706492f

25. Wang, C.; Chen, Q.; Sun, F.; Zhang, D.; Zhang, G.; Huang, Y.; Zhao, R.; Zhu, D. J. Am. Chem. Soc. 2010, 132, 3092-3096. doi:10.1021/ja910721s

26. Wu, Y.; Wu, S.; Tian, X.; Wang, X.; Wu, W.; Zou, G.; Zhang, Q. Soft Matter 2011, 7, 716-721. doi:10.1039/C0SM00330A

27. Wang, J.; Yang, G.; Jiang, H.; Zou, G.; Zhang, Q. Soft Matter 2013, 9 , 9785-9791. doi:10.1039/c3sm51896e

28. Gao, J.; He, Y.; Liu, F.; Zhang, X.; Wang, Z.; Wang, X. Chem. Mater. 2007, 19, 3877-3881. doi:10.1021/cm0707197

29. Bajaj, A.; Kondaiah, P.; Bhattacharya, S. Bioconjugate Chem. 2008, 19, 1640-1651. doi:10.1021/bc700381v

\section{License and Terms}

This is an Open Access article under the terms of the Creative Commons Attribution License

(http://creativecommons.org/licenses/by/2.0), which permits unrestricted use, distribution, and reproduction in any medium, provided the original work is properly cited.

The license is subject to the Beilstein Journal of Organic Chemistry terms and conditions:

(http://www.beilstein-journals.org/bjoc)

The definitive version of this article is the electronic one which can be found at: doi:10.3762/bjoc.11.122 